\title{
LAGRANGIAN EMBEDDINGS INTO SUBCRITICAL STEIN MANIFOLDS*
}

\author{
BY \\ PAUL Biran \\ School of Mathematical Sciences, Tel-Aviv University \\ Ramat-Aviv, Tel-Aviv 69978, Israel \\ e-mail: biran@math.tau.ac.il \\ AND \\ Kai CieliebaK \\ Department of Mathematics, Stanford University \\ Stanford, CA 94305-2125, USA \\ e-mail: kai@math.stanford.edu
}

\section{Introduction}

This paper is devoted to topological restrictions on Lagrangian embeddings. We extend results of Oh, Polterovich and Viterbo to the more general framework of "subcritical Stein manifolds".

The study of restrictions on Lagrangian embeddings began with Gromov's 1985 paper [Gr], in which he proved that $\mathbb{C}^{m}$ admits no closed exact Lagrangian submanifold. Later on, Polterovich [P] and Viterbo [V-1] found restrictions on the

* Research partially supported by the US-Israel Binational Science Foundation grant 1999086. 
Maslov class of closed Lagrangian submanifolds in $\mathbb{C}^{n}$, Polterovich for monotone Lagrangians, and Viterbo for Lagrangians that admit a metric of non-positive curvature. It is interesting to note that these results of the same nature were obtained by very different methods: Viterbo used Hamiltonian dynamics, whereas Polterovich used pseudo-holomorphic curves. Polterovich's result was generalized to split symplectic manifolds of the form $X \times \mathbb{C}$ in $[\mathrm{A}-\mathrm{L}-\mathrm{P}]$. The restriction found by Polterovich and Viterbo is that closed Lagrangian submanifolds of $\mathbb{C}^{n}$ (of the types just mentioned) have minimal Maslov number between 1 and $n+1$. Viterbo's result gives an affirmative answer, in dimension 4, to Audin's Conjecture [A] that all Lagrangian tori in $\mathbb{C}^{n}$ have minimal Maslov number 2.

Monotone Lagrangian embeddings into $\mathbb{C}^{n}$ were later studied by $\mathrm{Oh}$ [Oh-3] using the powerful technique of Floer homology. In particular, he confirmed Audin's conjecture for the case of monotone tori in $\mathbb{C}^{n}$ for $n \leq 24$. Recently, Viterbo [V-2] applied Floer homology to obtain restrictions on the Maslov class on subcritical Stein manifolds.

In this paper we extend some of the above results to a more natural class of manifolds called "subcritical split manifolds" (see Section 2). The main tools we use are a combination of Oh's analysis of the space of solutions to Floer's perturbed Cauchy-Riemann equation corresponding to isotopies of Lagrangian submanifolds [Oh-4], and some ideas developed by Polterovich in [P]. These techniques, as it will turn out below, work particularly well in the framework of subcritical split manifolds.

As application we obtain new restrictions on Lagrangian embeddings of manifolds $L$ with $H_{1}(L, \mathbb{Z})=0$ into subcritical split manifolds.

Further applications will be given in a forthcoming paper [B-C] in which Lagrangian embeddings into closed manifolds will be studied.

STEIN MANifolds And domains. A Stein manifold is a triple $(V, J, \varphi)$ where $(V, J)$ is an open complex manifold and $\varphi: V \rightarrow \mathbb{R}$ is a smooth exhausting plurisubharmonic function. The term "exhausting" means that $\varphi$ is proper and bounded from below. "Plurisubharmonic" means that the 2 -form $\omega_{\varphi}=-d d^{\mathbb{C}} \varphi$ is a $J$-positive symplectic form, i.e., $-d d^{\mathbb{C}}(v, J v)>0$ for every $0 \neq v \in T V$. We denote by $g_{\varphi}(\cdot, \cdot)=\omega_{\varphi}(\cdot, J)$ the associated Kähler Riemannian metric. Unless explicitly stated, we do not assume that $(V, J, \varphi)$ is complete in the EliashbergGromov [E-G] sense.

A Stein domain is a subset $V_{\varphi<c}=\{p \in V \mid \varphi(p)<c\}$ of a Stein manifold $(V, J, \varphi)$ for a regular value $c \in \mathbb{R}$. We will often denote Stein domains simply by $V$. 
SUbCRITICAL Stein MANifolds. It is well known that any plurisubharmonic Morse function $\varphi: V \rightarrow \mathbb{R}$ must satisfy index $\operatorname{dex}_{p}(\varphi) \leq \frac{1}{2} \operatorname{dim}_{\mathbb{R}} V$ for all critical points $p$. We call a Stein manifold/domain subcritical if these inequalities are strict, i.e., $\varphi$ is a Morse function all of whose critical points have $\operatorname{index}_{p}(\varphi)<$ $\frac{1}{2} \operatorname{dim}_{\mathbb{R}} V$. Note that, by definition, 0 -dimensional manifolds (namely points) are not subcritical.

The simplest subcritical Stein manifold is $\mathbb{C}^{k}$ endowed with its standard complex structure and the plurisubharmonic function $\varphi\left(z_{1}, \ldots, z_{n}\right)=\left|z_{1}\right|^{2}+\cdots+$ $\left|z_{n}\right|^{2}$. Note that the class of subcritical Stein manifolds is closed under several simple constructions such as taking products with any Stein manifold, attaching handles of index smaller than half of the dimension etc.

It should also be remarked that in view of Eliashberg's theory of Stein manifolds [E], subcriticality is essentially a topological condition in dimension 6 and above.

Applications to Lagrangian embeddings With $H_{1}(L ; \mathbb{Z})=0$. The following two theorems illustrate some typical applications obtained from our main results in this paper. More general statements are given in Section 2.

The first theorem gives a restriction on the topology of manifolds that admit Lagrangian embeddings into products of subcritical Stein manifolds with $\mathbb{C} P^{n}$. Here and in what follows we endow $\mathbb{C} P^{n}$ with its standard symplectic structure $\sigma_{\text {std }}$, normalized so that the area of a projective line is $\pi$.

THEOREM 1.1: Let $(V, J, \varphi)$ be a subcritical Stein manifold with $\operatorname{dim}_{\mathbb{R}} V \geq 2$ and whose first Chern class (of its tangent bundle) vanishes on $\pi_{2}(V)$. Let $n \geq$ $\frac{1}{2} \operatorname{dim}_{\mathbb{R}} V-1$. Then every Lagrangian submanifold $L \subset\left(V \times \mathbb{C} P^{n}, \omega_{\varphi} \oplus \sigma_{\text {std }}\right)$ with $H_{1}(L ; \mathbb{Z})=0$ must satisfy

$$
H^{1}\left(L ; \mathbb{Z}_{2}\right)=\cdots=H^{\operatorname{dim} L-1}\left(L ; \mathbb{Z}_{2}\right)=0 .
$$

Note that the statement of Theorem 1.1 is not empty. Indeed, the manifold $\mathbb{C}^{n+1} \times \mathbb{C} P^{n}$ has a Lagrangian $(2 n+1)$-sphere $L$ constructed as follows (see [A-L-P]). Denote by $S^{2 n+1} \subset \mathbb{C}^{n+1}$ the unit sphere and by $h: S^{2 n+1} \rightarrow \mathbb{C} P^{n}$ the Hopf map. Then

$$
L=\left\{(\bar{z}, h(z)) \mid z \in S^{2 n+1}\right\} \subset \mathbb{C}^{n+1} \times \mathbb{C} P^{n}
$$

is a Lagrangian sphere.

The second theorem shows that Lagrangian submanifolds with vanishing first homology do not exist at all if the size of the Stein factor is sufficiently small. 
THEOREM 1.2: Let $\left(V_{\varphi<c}, J, \varphi\right)$ be a subcritical Stein domain with $\operatorname{dim} V>0$. Then there exists an $\alpha_{0}>0$ such that for every closed symplectic manifold $\left(X, \omega_{X}\right)$ with $\left[\omega_{X}\right] \in H^{2}(X ; \mathbb{Z})$ and for every $0<\alpha \leq \alpha_{0},\left(V_{\varphi<c} \times X, \alpha \omega_{\varphi} \oplus \omega_{X}\right)$ does not admit any Lagrangian submanifold $L$ with $H_{1}(L ; \mathbb{Z})=0$.

Note that if $X$ is taken to be a point then the theorem is true without the rescaling factor. The existence of Lagrangian spheres in $\mathbb{C} P^{n} \times \mathbb{C}^{n+1}$ (discussed after Theorem 1.1) shows that in general Theorem 1.2 is false without the scaling factor $\alpha$. In this example, for $V_{\varphi<1}=\operatorname{Int} B^{2 n}(1)$, we can choose $\alpha_{0}=1$ (see Section 2).

Other examples in which the theorem fails without the factor $\alpha$ come from [Bi]. Consider $X \subset \mathbb{C} P^{n} \times \mathbb{C} P^{n+r}(n \geq 1, r \geq 0)$ with homogeneous coordinates $\left[z_{0}: \ldots: z_{n}\right],\left[w_{0}: \ldots: w_{n+r}\right]$ and symplectic form $\omega=\sigma_{\text {std }} \oplus \sigma_{\text {std }}$. Let $X$ be the hypersurface given by the equation

$$
X=\left\{\sum_{i=0}^{n-1} z_{i} w_{i}=z_{n} \sum_{j=1}^{n+r} w_{j}\right\} .
$$

The complement $V=\mathbb{C} P^{n} \times \mathbb{C} P^{n+r} \backslash X$ is a subcritical Stein manifold (of finite volume) in a natural way. Let $L$ be circle bundle over $X$ obtained as the boundary of a tubular neighbourhood of $X$ in $\mathbb{C} P^{n} \times \mathbb{C} P^{n+r}$. Then $H_{1}(L ; \mathbb{Z})=0$, and $L$ has a Lagrangian embedding into $\left(V \times X,\left.\left.\beta \omega\right|_{V} \oplus \omega\right|_{X}\right)$ for every $\beta>1$.

\section{Main results}

SUBCRITICAL SPLIT MANIFOLDS. We say that a product $W=V \times X, \omega_{W}=$ $\omega_{\varphi} \oplus \omega_{X}, J_{W}=J_{V} \oplus J_{X}$ is subcritical split if

- $\left(V, J_{V}, \varphi\right)$ is either a subcritical Stein manifold or a subcritical Stein domain. We require that $\operatorname{dim} V>0$.

- $\left(X, \omega_{X}, J_{X}\right)$ is either geometrically bounded in the sense of [A-L-P] or convex at infinity in the sense of [E-G].

We allow $X$ to be zero dimensional. The class of manifolds $X$ satisfying the hypothesis includes: closed symplectic manifolds, Stein manifolds / domains, and interiors of compact symplectic manifolds with $J_{X}$-convex boundary.

MASLOV CLASS RIGIDITY. We start with a generalization of results by Polterovich [P, A-L-P] and Viterbo [V-2].

Consider a Lagrangian submanifold $L$ of a symplectic manifold $(M, \omega)$. The Maslov index $\mu: \pi_{2}(M, L) \rightarrow \mathbb{Z}$ is defined as follows: Let $D \subset M$ be a disk with $\partial D \subset L$. Symplectically trivialize the bundle $\left.T M\right|_{D}$. The image of the 
subbundle $\left.T L\right|_{\partial D}$ under the trivialization is a loop of Lagrangian subspaces of $\mathbb{C}^{n}$ whose homotopy class depends only on $[D] \in \pi_{2}(M, L)$. Define $\mu([D])$ to be the Maslov index of this loop (see [M-S]).

Given a symplectic manifold $(M, \omega)$, we denote by $c_{1}^{M} \in H^{2}(M ; \mathbb{Z})$ the first Chern class of $T M$ viewed as a complex vector bundle (by endowing it with any $\omega$-compatible almost complex structure).

Theorem 2.1: Let $\left(W=V \times X, \omega_{W}, J_{W}\right)$ be a subcritical split manifold and $L \subset W$ a closed Lagrangian submanifold. Then at least one of the following two statements holds:

1. There exists a nonconstant reduced $J_{W}$-holomorphic disk $D \subset W$ with $\partial D \subset L$ and

$$
\mu([D]) \leq \operatorname{dim} L+1 .
$$

2. There exists a nonconstant reduced $J_{W}$-holomorphic sphere $S \subset W$ with

$$
2 c_{1}^{W}([S]) \leq \operatorname{dim} L+1
$$

Remarks:

1. In case $\left(X, \omega_{X}\right)$ is exact it immediately follows that the Lagrangian $L$ cannot be exact. For $X=p t$ and $V=\mathbb{C}^{n}$ this is Gromov's celebrated result [Gr]. It was generalized in $[\mathrm{A}-\mathrm{L}-\mathrm{P}]$ to $X, L$ geometrically bounded and $V=\mathbb{C}$.

2. For $X=\mathrm{pt}$ and $\left.c_{1}^{V}\right|_{\pi_{2}(V)}=0$, Theorem 2.1 has been proved by Viterbo in $[\mathrm{V}-2]$.

3. If $L$ is monotone (see below) the positivity of $\omega$ on holomorphic curves implies $\mu([D]) \geq 1$ and $2 c_{1}^{W}([S]) \geq 2$. For monotone $L$, Theorem 2.1 has been proved in [A-L-P] under the assumptions $X, L$ geometrically bounded, $\left.\omega_{X}\right|_{\pi_{2}(X)}=0$ and $V=\mathbb{C}$. The case $L$ monotone, $X=\mathrm{pt}$ and $V=\mathbb{C}^{n}$ was proved in $[\mathrm{P}]$.

4. The theorem remains true if $J_{W}$ is replaced by any $\omega_{W}$-compatible almost complex structure $J$ that coincides with $J_{W}$ outside a compact set.

5. If $J_{W}$ is replaced by a generic $J$ as in 4 . we also get the lower bound

$$
\mu([D]) \geq 3-\operatorname{dim} L .
$$

This will be shown in the proof of Theorem 2.1.

ShARPER Bounds For MONOTONE MANIfOlds. Using the method in [Oh-3], we can replace the upper bound $\operatorname{dim} L+1$ by $\operatorname{dim} L$ for monotone manifolds.

A symplectic manifold $(M, \omega)$ is called monotone (resp. spherically monotone) if $[\omega]=\lambda c_{1}^{M}$ in $H^{2}(M ; \mathbb{R})$ (resp. $[\omega]=\lambda c_{1}^{M}$ on $\pi_{2}(M)$ ) for some constant 
$\lambda>0$. A Lagrangian submanifold $L \subset(M, \omega)$ is called monotone if $[\omega]=\lambda \mu$ on $\pi_{2}(M, L)$ for some constant $\lambda>0$ (cf. [Oh-2]). Note that if $(M, \omega)$ admits a monotone Lagrangian submanifold then $(M, \omega)$ is automatically spherically monotone.

THEOREM 2.2: Let $\left(W=V \times X, \omega_{W}, J_{W}\right)$ be a subcritical split manifold and $L \subset W$ a closed Lagrangian submanifold. Assume in addition that $L$ is monotone and $\operatorname{dim}_{\mathbb{R}} W \geq 4$. Then at least one of the following three statements holds:

1. There exists a nonconstant reduced $J_{W}$-holomorphic disk $D \subset W$ with $\partial D \subset L$ and

$$
\mu([D]) \leq \operatorname{dim} L .
$$

2. There exists a nonconstant reduced $J_{W}$-holomorphic sphere $S \subset W$ with

$$
2 c_{1}^{W}([S]) \leq \operatorname{dim} L
$$

3. $H^{1}\left(L ; \mathbb{Z}_{2}\right)=\cdots=H^{\operatorname{dim} L-1}\left(L ; \mathbb{Z}_{2}\right)=0$.

The following corollary generalizes a result due to $\mathrm{Oh}[\mathrm{Oh}-3]$ in the case $X=\mathrm{pt}$ and $V=\mathbb{C}^{n}(n \geq 2)$.

Corollary 2.3: Let $W$ and $L$ be as in Theorem 2.2. Assume further that $\left(X, \omega_{X}\right)$ is exact. Then there exists a nonconstant reduced $J$-holomorphic disk $D \subset W$ with $\partial D \subset L$ and such that

$$
1 \leq \mu([D]) \leq \operatorname{dim} L
$$

Proof: Since both $\left(V, \omega_{\varphi}\right)$ and $\left(X, \omega_{X}\right)$ are exact so is also $\left(W, \omega_{W}\right)$, hence it does not admit any $J$-holomorphic spheres. By Theorem 2.1 there exists a $J$ holomorphic disk in $W$ with boundary on $L$. As $\left(W, \omega_{W}\right)$ is exact this implies that $H^{1}(L, \mathbb{Z}) \neq 0$. In particular $H^{1}\left(L ; \mathbb{Z}_{2}\right) \neq 0$ and so the existence of the desired disk follows from Theorem 2.2.

Remark: The upper bound in Corollary 2.3 cannot in general be improved: Polterovich ([P], based on constructions in $[\mathrm{A}]$ ) constructed closed monotone submanifolds of $\mathbb{C}^{n}$ of minimal Maslov index $k$ for any $2 \leq k \leq n$.

RESTRICTIONS ON HOMOLOGY. Now we describe some assumptions on the symplectic manifold under which the first two cases of Theorem 2.2 cannot occur, thus obtaining restrictions on the homology of a Lagrangian submanifolds.

Given a monotone symplectic manifold $(M, \omega)$ denote

$$
N_{M}^{H_{2}}=\min \left\{c_{1}^{M}(B) \mid B \in H_{2}(M ; \mathbb{Z}) \text { and } \omega(B)>0\right\}
$$


Similarly if $(M, \omega)$ is spherically monotone we write

$$
N_{M}^{\pi_{2}}=\min \left\{c_{1}^{M}(B) \mid B \in \pi_{2}(M) \text { and } \omega(B)>0\right\} .
$$

Corollary 2.4: Assume that $\left(W=V \times X, \omega_{W}, J_{W}\right)$ is subcritical split and satisfies the following two conditions:

1. $c_{1}^{V}=0$ in $H^{2}(V ; \mathbb{Q})$ (resp. $\left.c_{1}^{V}\right|_{\pi_{2}(V)}=0$ );

2. $\left(X, \omega_{X}\right)$ is monotone (resp. spherically monotone) with $2 N_{X}^{H_{2}}>\frac{1}{2} \operatorname{dim}_{\mathbb{R}} W$ (resp. $2 N_{X}^{\pi_{2}}>\frac{1}{2} \operatorname{dim}_{\mathbb{R}} W$ ).

Then any closed Lagrangian submanifold $L \subset\left(W, \omega_{W}\right)$ with $H_{1}(L ; \mathbb{Z})=0$ (resp. $L$ simply connected) must satisfy $H^{1}\left(L ; \mathbb{Z}_{2}\right)=\cdots=H^{\operatorname{dim} L-1}\left(L ; \mathbb{Z}_{2}\right)=0$.

Proof: For $\operatorname{dim}_{\mathbb{R}} W=2$ there exists no closed (Lagrangian sub-) manifold with $H_{1}(L ; \mathbb{Z})=0$, so assume $\operatorname{dim}_{\mathbb{R}} W \geq 4$. The assumptions of the corollary imply that $H_{2}(W ; \mathbb{Z}) \rightarrow H_{2}(W, L ; \mathbb{Z})$ (resp. $\left.\pi_{2}(W) \rightarrow \pi_{2}(W, L)\right)$ is surjective. Therefore the assumption on $c_{1}^{V}$ and the monotonicity of $\left(X, \omega_{X}\right)$ imply that $L \subset\left(W, \omega_{W}\right)$ is monotone. Finally, from the inequality on $N_{X}^{H_{2}}$ (resp. $N_{X}^{\pi_{2}}$ ) we see that the first two possibilities in Theorem 2.2 cannot occur. Therefore, $H^{1}\left(L ; \mathbb{Z}_{2}\right)=\cdots=$ $H^{\operatorname{dim} L-1}\left(L ; \mathbb{Z}_{2}\right)=0$.

Proof of Theorem 1.1: Theorem 1.1 follows immediately from Corollary 2.4 and $N_{C P^{n}}^{H_{2}}=n+1$.

RESTRICTIONS COMING FROM DISPLACEMENT ENERGY. In Section 3 we define the displacement energy $0<e\left(V, J_{V}, \varphi\right)<\infty$ of a subcritical Stein domain. It has the scaling property

$$
e\left(V, J_{V}, \lambda \varphi\right)=\lambda e\left(V, J_{V}, \varphi\right) \text { for } \lambda>0 .
$$

THEOREM 2.5: Let $\left(W=V \times X, \omega_{W}, J_{W}\right)$ be subcritical split, where $V$ is a subcritical Stein domain with displacement energy $e\left(V, J_{V}, \varphi\right)$. Then the $J_{W}$ holomorphic disks $D$ and spheres $S$ in all previous results may be assumed to satisfy

$$
0<\omega([D]), \omega([S]) \leq e\left(V, J_{V}, \varphi\right)
$$

Corollary 2.6: Let $\left(W=V \times X, \omega_{W}, J_{W}\right)$ be subcritical split. Assume that $\left[\omega_{X}\right] \in H^{2}(X ; \mathbb{Z})$ is an integral class, and $V$ is a subcritical Stein domain with displacement energy $e\left(V, J_{V}, \varphi\right)<1$.

Then $\left(W, \omega_{w}\right)$ has no closed Lagrangian submanifold $L$ with $H_{1}(L ; \mathbb{Z})=0$.

Proof: In view of the hypotheses of the corollary and Theorem 2.5, JWholomorphic spheres cannot occur in the conclusion of Theorem 2.1. So there 
exists a $J_{W}$-holomorphic disk $(D, \partial D) \subset(W, L)$ with $0<\omega_{W}([D])<1$. If $H_{1}(L ; \mathbb{Z})=0, \partial D$ bounds a surface $\Sigma \subset L$. But then $0<\omega_{W}\left(\left[D \cup_{\partial D} \Sigma\right]\right)<1$, contradicting the integrality of $\left[\omega_{X}\right]$.

Remark: Corollary 2.6 remains true if the integrality of $\left[\omega_{X}\right]$ is replaced by the weaker hypothesis

$$
\lambda:=\inf \left\{\omega_{X}(\alpha) \mid \alpha \in H_{2}(X ; \mathbb{Z}), \omega_{X}(\alpha)>0\right\}>0
$$

and $e\left(V, J_{V}, \varphi\right)<1$ is replaced by $e\left(V, J_{V}, \varphi\right)<\lambda$.

Proof of Theorem 1.2: Simply choose $\alpha=e\left(V, J_{V}, \phi\right)^{-1}$.

For example, let $B^{2 m}(r)$ be the closed $2 m$-dimensional ball of radius $r$.

CORollaRY 2.7: For $m \geq 1$ and $r<1, \mathbb{C} P^{n} \times B^{2 m}(r)$ has no closed Lagrangian submanifold $L$ with $H_{1}(L ; \mathbb{Z})=0$.

Proof: This follows directly from Corollary 2.6 because the symplectic form $\frac{\sigma_{\text {std }}}{\pi}$ on $\mathbb{C P}^{n}$ is integral, and $e\left(B^{2 m}(r)\right)=\pi r^{2}$ by a result of Hofer $[\mathrm{H}-\mathrm{Z}]$ (in fact, here we only need the easy inequality $\left.e\left(B^{2 m}(r)\right) \leq \pi r^{2}\right)$.

Remarks:

1. For $m=n+1$ and $r>1, \mathbb{C} P^{n} \times B^{2 m}(r)$ has the Lagrangian $(2 n+1)$-sphere described in the remark following Theorem 1.1.

2. For $m>n+1$ no examples are known to us.

NON-LINKING WITH HYPERSURFACES. Here we generalize a result of Mohnke $[\mathrm{M}]$ on the non-linking of Lagrangian submanifolds with complex hypersurfaces.

In analogy to algebraic geometry we call a (properly embedded and without boundary) complex hypersurface $\Sigma \subset\left(X, J_{X}\right)$ of an almost complex manifold spherically nef ("nef" stands for "numerically effective") if for every $J_{X}$ holomorphic sphere $C \subset\left(X, J_{X}\right), \Sigma \cdot C \geq 0$. Of course, if $\left(X, J_{X}\right)$ has no $J_{X}$-holomorphic spheres then any hypersurface $\Sigma$ is automatically nef.

THEOREM 2.8: Let $\left(W=V \times X, \omega_{W}, J_{W}\right)$ be subcritical split and $L \subset W$ a closed Lagrangian manifold. Let $\Sigma \subset X$ be a properly embedded $J_{X}$-complex hypersurface which is spherically nef and lies in the complement to $\mathrm{pr}_{X}(L)$.

Then the $J_{W}$-holomorphic disks $D$ and spheres $S$ in all previous results may be assumed to lie in the complement of $V \times \Sigma$.

This implies the following generalization of Mohnke's result in [M]. 
Corollary 2.9: Let $\left(W=V \times X, \omega_{W}, J_{W}\right)$ be subcritical split. Let $\Sigma \subset$ $\left(X, J_{X}\right)$ be a properly embedded $J_{X}$-complex hypersurface which is spherically nef. Let $L \subset\left(W, \omega_{W}\right)$ be a Lagrangian submanifold which lies in the complement of $V \times \Sigma$ in $W$.

Then there exists a disk $(D, \partial D) \subset(W, L)$ in the complement of $V \times \Sigma$ with $\omega_{W}([D])>0$. In particular, if $\operatorname{dim} H_{2}(W, L ; \mathbb{Q})=1$ the linking homomorphism

$$
\ell k_{L, V \times \Sigma}: H_{2}(W, L ; \mathbb{Z}) \rightarrow \mathbb{Z}
$$

(defined by intersecting with $V \times \Sigma$ ) vanishes.

Proof: By Theorem 2.8 there exists in the complement of $V \times \Sigma$ either a nonconstant $J_{W}$-holomorphic disk $D$ with boundary on $L$, or a nonconstant $J_{W}$-holomorphic sphere $S$. In case of a disk, denote by $A \in H_{2}(W, L ; \mathbb{Z})$ its relative homology class. In case of a sphere $S$, put $A$ to be the image of $[S] \in H_{2}(W ; \mathbb{Z})$ under the homomorphism $H_{2}(W ; \mathbb{Z}) \rightarrow H_{2}(W, L ; \mathbb{Z})$. Since $\omega(A)>0, A \in H_{2}(W, L ; \mathbb{Z})$ is not torsion. So $\ell k_{L, V \times \Sigma}(A)=0$ implies that $\ell k_{L, V \times \Sigma}$ vanishes on the non-torsion subgroup, and therefore vanishes altogether.

Remark: In the case $X=V=\mathbb{C}$ and $\Sigma=$ pt, Corollary 2.9 easily implies that a Lagrangian embedding of the Klein bottle into $\mathbb{C}^{2}$, if it exists, must be homologically unlinked with every real plane of dimension 2 (see [M]).

Discussion. Let us briefly explain the main geometric ideas behind the proofs of the theorems above. The main phenomenon standing behind most of our result is the breakdown of the symplectic principle of Lagrangian intersections.

Since the beginning of symplectic topology it was conjectured that in some situations a Lagrangian submanifold cannot be disjoined from itself via a Hamiltonian isotopy. This was indeed confirmed in many cases (such as the zero section in cotangent bundles) via various methods and techniques, most notably pseudoholomorphic curves (see $[\mathrm{Gr}]$ ).

An important and conceptual progress in understanding the nature of these intersections was made with the introduction of Floer homology [F-2]. This homology theory detects (and counts) intersections between pairs of Hamiltonian isotopic Lagrangians $L$ and $h(L)$. Floer proved [F-2] that essentially the only thing that might fail this invariant to detect intersections is the existence of pseudo-holomorphic disks (or spheres) with boundary on the Lagrangian $L$ occurring in a process called bubbling (see [Ch] for a more delicate analysis of the situation). 
When bubbling does occur, these disks come in finite dimensional families and it is possible to estimate their dimensions in terms of the Maslov index. Consequently, whenever it is possible to disjoin a Lagrangian submanifold from itself via a Hamiltonian isotopy one obtains restrictions on the Maslov index corresponding to this Lagrangian as in Theorem 2.1.

The key point leading to our main results is that due to their special symplectic nature, subcritical split manifolds allow to disjoin every Lagrangian submanifold from itself via a Hamiltonian isotopy. In other words, the (global) phenomenon of Lagrangian intersections never occurs in this class of manifolds.

The rest of the paper is organized as follows. In Section 3 we prove that every compact subset of a subcritical Stein manifold can be disjoined from itself by a Hamiltonian isotopy, and define the displacement energy of a Stein domain. Section 4 contains the proof of the theorems. We study the space of solutions of a perturbed Cauchy-Riemann equation as in [Oh-4], and show how bubbling leads to the proof of Theorem 2.1. Theorem 2.2 is based on the vanishing of Floer homology, Theorem 2.5 on an area estimate for holomorphic curves, and Theorem 2.8 on positivity of intersections of complex submanifolds.

ACKNOWLEDGEMENT: We wish to thank Laurent Lazzarini for sending us his manuscript [La] and for useful explanations on reduction of holomorphic disks to simple disks. We also thank the referee for several useful comments that improved the quality of the exposition.

Part of this work was carried out during the second author's visit to TelAviv University in June 2000. He would like to thank the Minkowski Centre for Geometry for supporting this visit.

\section{Hamiltonian translations on subcritical Stein manifolds}

Following $[\mathrm{E}-\mathrm{G}]$ we say that a Stein manifold $(V, J, \varphi)$ is complete if the flow of gradient vector field $X_{\varphi}=\operatorname{grad}_{g_{\varphi}} \varphi$ exists for all positive times.

LEMma 3.1 (Completion [E-G]): Let $(V, J, \varphi)$ be a Stein manifold. Then for every $R \in \mathbb{R}$ there exists an exhausting plurisubharmonic function $\varphi_{R}: V \rightarrow \mathbb{R}$ with the following properties:

1. $\varphi_{R}=\varphi$ on $V_{\varphi \leq R}$.

2. $\left(V, J, \varphi_{R}\right)$ is a complete Stein manifold.

3. $\operatorname{Crit}\left(\varphi_{R}\right)=\operatorname{Crit}(\varphi)$ and for every $p \in \operatorname{Crit}\left(\varphi_{R}\right), \operatorname{index}_{p}\left(\varphi_{R}\right)=\operatorname{index}_{p}(\varphi)$. In particular, the inclusion $\left(V_{\varphi \leq R}, \omega_{\varphi}\right) \subset\left(V, \omega_{\varphi_{R}}\right)$ is a symplectic embedding. 
Proof: Let $h: \mathbb{R} \rightarrow \mathbb{R}$ be a smooth function with $h^{\prime}>0, h^{\prime \prime} \geq 0$, and $h(r)=r$ for $r \leq R$. Set $\varphi_{R}:=h \circ \varphi$. Then

$$
\omega_{\varphi_{R}}=h^{\prime} \omega_{\varphi}+h^{\prime \prime} d \varphi \wedge \lambda_{\varphi}, \quad X_{\varphi_{R}}=\frac{h^{\prime}}{h^{\prime}+\left|X_{\varphi}\right|^{2} h^{\prime \prime}} X_{\varphi}
$$

The first formula shows that $\varphi_{R}$ is plurisubharmonic. Now assume, in addition, that $h^{\prime \prime}(r) \geq h^{\prime}(r)$ for $r \geq R+1$. Then by the second formula, $\left|X_{\varphi_{R}}\right| \leq 1$ for $r \geq R+1$. Denote by $X_{\varphi_{R}}^{t}$ the flow of $X_{\varphi_{R}}$. Since

$$
\frac{d}{d t} \varphi_{R} \circ X_{\varphi_{R}}^{t}(x)=\left|X_{\varphi_{R}}\right|^{2} \leq 1
$$

whenever $\varphi \circ X_{\varphi_{R}}^{t}(x) \geq R+1$, the function $\varphi \circ X_{\varphi_{R}}^{t}(x)$ grows at most linearly as $t \rightarrow \infty$, so the flow $X_{\varphi_{R}}^{t}$ is complete.

An explicit example of a function $h$ satisfying all the conditions is

$$
\begin{cases}h(r)=r & \text { for } r \leq R \\ h^{\prime \prime}(r) \geq 0 & \text { for } R \leq r \leq R+1 \\ h(r)=e^{a r} & \text { for } r \geq R+1\end{cases}
$$

with $a \geq 1$.

Lemma 3.2 (Hamiltonian translations): Let $(V, J, \varphi)$ be a complete subcritical Stein manifold. Then for every compact subset $A \subset V$ there exists a compactly supported Hamiltonian isotopy $h_{t}:\left(V, \omega_{\varphi}\right) \rightarrow\left(V, \omega_{\varphi}\right)$ such that $h_{1}(A) \cap A=\emptyset$.

Proof: Denote by $\Delta_{\varphi} \subset V$ the union of the stable submanifolds of the gradient vector field $X_{\varphi}=\operatorname{grad}_{g_{\varphi}} \varphi$, namely

$$
\Delta_{\varphi}=\bigcup_{p \in \operatorname{Crit}(\varphi)} W_{p}^{s}\left(X_{\varphi}\right)
$$

We shall first prove the lemma under the assumption that $\Delta_{\varphi}$ is a CW-complex, and then show how to reduce the general case to this assumption.

Suppose indeed that $\Delta_{\varphi}$ is a CW-complex. Since $V$ is subcritical we have $\operatorname{dim} \Delta_{\varphi}<\frac{1}{2} \operatorname{dim}_{\mathbb{R}} V$ and so there exists a Hamiltonian isotopy $k_{t}:\left(V, \omega_{\varphi}\right) \rightarrow$ $\left(V, \omega_{\varphi}\right)$, compactly supported in an arbitrarily small neighbourhood of $\Delta_{\varphi}$, such that $k_{1}\left(\Delta_{\varphi}\right) \cap \Delta_{\varphi}=\emptyset$. Since $\Delta_{\varphi}$ is compact there exists a small neighbourhood $U$ of $\Delta_{\varphi}$ so that $k_{1}(U) \cap U=\emptyset$.

Denote by $X_{\varphi}^{t}(t \in \mathbb{R})$ the flow of $X_{\varphi}$. Fix $T$ so large that $X_{\varphi}^{-T}(A) \subset U$. Since $k_{1}$ moves $U$ away from itself we have

$$
X_{\varphi}^{T} \circ k_{1} \circ X_{\varphi}^{-T}(A) \cap A=\emptyset .
$$


Since $L_{X_{\varphi}} \omega_{\varphi}=\omega_{\varphi}$, the flow $X_{\varphi}^{t}$ is conformally symplectic, $\left(X_{\varphi}^{t}\right)^{*} \omega_{\varphi}=e^{t} \omega_{\varphi}$. This implies that

$$
h_{t}=X_{\varphi}^{t T_{0}} \circ k_{t} \circ X_{\varphi}^{-t T_{0}}
$$

is a symplectic isotopy of $\left(V, \omega_{\varphi}\right)$ with $h_{1}(A) \cap A=\emptyset$. A straightforward calculation shows that $h_{t}$ is generated by the compactly supported time-dependent Hamiltonian

$$
H_{t}=e^{t T}\left(X_{\varphi}^{-t T}\right)^{*}\left(K_{t}+G_{t}\right)
$$

Here $K_{t}$ is a compactly supported Hamiltonian generating the isotopy $k_{t}$. $G_{t}$ is the unique compactly supported function satisfying

$$
d G_{t}=\lambda_{\varphi}-k_{-t}^{*} \lambda_{\varphi}
$$

for every $t$, where $\lambda_{\varphi}=-d^{\mathbb{C}} \varphi$. The function $G_{t}$ is given explicitly by

$$
G_{t}(x)=\int_{0}^{t}\left(K_{s}-d K_{s}\left(X_{\varphi}\right)\right) k_{-s}(x) d s .
$$

It remains to show how to reduce the general case to a situation in which $\Delta_{\varphi}$ is a CW-complex. By the results of [Bi] (see Theorem 8.1.B there), there exists a plurisubharmonic Morse function $\varphi^{\prime}: V \rightarrow \mathbb{R}$ that coincides with $\varphi$ on $\{\varphi \geq R\}$ for some $R$ and such that $\Delta_{\varphi^{\prime}}$ is a CW-complex of the same dimension as the cellular space $\Delta_{\varphi}$, namely

$$
\operatorname{dim} \Delta_{\varphi^{\prime}}=\max _{p \in \operatorname{Crit}(\varphi)} \operatorname{index}_{p}(\varphi)<\frac{1}{2} \operatorname{dim}_{\mathbb{R}} V
$$

Since $\varphi^{\prime}$ and $\varphi$ are both plurisubharmonic functions for the same complex structure $J$ and coincide at "infinity" it follows from Moser's argument that $\omega_{\varphi}$ is symplectomorphic to $\omega_{\varphi^{\prime}}$ via a compactly supported diffeomorphism of $V$.

Thus replacing $\varphi$ by $\varphi^{\prime}$ we may assume that $\Delta_{\varphi}$ is a CW-complex.

Following Hofer (cf. [H-Z]), define the norm of a compactly supported timedependent Hamiltonian $H(t, x)$ on a symplectic manifold $(M, \omega)$ as

$$
\|H\|:=\int_{0}^{1}\left(\max _{x \in M} H(t, x)-\min _{x \in M} H(t, x)\right) d t .
$$

Define the displacement energy of a subset $A \subset M$ as

$$
e(A):=\inf _{H}\left\{\|H\| h_{1}(A) \cap A=\emptyset\right\},
$$

where $h_{1}$ is the time-1 map of the Hamiltonian flow of $H$, and the infimum is taken over all compactly supported time-dependent Hamiltonians. 
Lemma 3.2 can be restated as saying that every compact subset of a complete subcritical Stein manifold has finite displacement energy.

Now consider a subcritical Stein domain $\left(V=V_{\varphi<c}, J, \varphi\right)$. By Lemma 3.1, $V$ is contained in a complete subcritical Stein manifold $(\hat{V}, \hat{J}, \hat{\varphi})$ such that $\left.\hat{\varphi}\right|_{V}=\varphi$ and $\hat{\varphi}$ has no critical points outside $V$. Define the displacement energy $e(V, J, \varphi)$ to be the displacement energy of $V$ in $\left(\hat{V}, \omega_{\hat{\varphi}}\right)$. This definition is independent of the choice of the completion because any two completions $(\hat{V}, \hat{J}, \hat{\varphi})$, $(\tilde{V}, \tilde{J}, \tilde{\varphi})$ are symplectomorphic $[\mathrm{E}-\mathrm{G}]:$ For every $n \in \mathbb{N}$ pick a constant $T_{n}$ such that

$$
X_{\hat{\varphi}}^{-T_{n}}\left(\hat{V}_{\hat{\varphi} \leq n}\right) \subset V
$$

and define

$$
f_{n}:=X_{\hat{\varphi}}^{T_{n}} \circ X_{\hat{\varphi}}^{-T_{n}} \text { on } \hat{V}_{\hat{p} \leq n}
$$

Since $f_{n}=f_{m}$ on $\hat{V}_{\hat{\varphi} \leq n} \cap \hat{V}_{\hat{\varphi} \leq m}$, these maps fit together to a symplectomorphism $f:\left(\hat{V}, \omega_{\hat{\varphi}}\right) \rightarrow\left(\tilde{V}, \omega_{\tilde{\varphi}}\right)$.

In view of Lemma 3.2 , the displacement energy of a subcritical Stein domain satisfies

$$
0<e(V, J, \varphi)<\infty
$$

It has the scaling property

$$
e(V, J, \lambda \varphi)=\lambda e(V, J, \varphi) \text { for } \lambda>0 .
$$

\section{Proof of Theorems $2.1,2.2,2.5$ and 2.8}

Proof of Theorem 2.1: The proof is based on ideas from [Oh-3] and [Oh-4].

Let $W=V \times X$ and $L$ be as in Theorem 2.1. Choose $c<\sup _{V} \varphi$ large enough so that $V_{\varphi<c} \times X$ contains $L$ and all the critical points of $\varphi$. Let $\phi=\varphi_{c}$ be the plurisubharmonic function obtained from Lemma 3.1, making $\left(V, J_{V}, \phi\right)$ a complete Stein manifold. Clearly $\left(V, J_{V}, \phi\right)$ is also subcritical. Put $\bar{W}=V \times X$, $J_{\bar{W}}=J_{V} \oplus J_{X}$ and $\omega_{\bar{W}}=\omega_{\phi} \oplus \omega_{X}$. Clearly, the inclusion

$$
\left(V_{\varphi<c} \times X, \omega_{w}=\omega_{\varphi} \oplus \omega_{X}\right) \subset\left(\bar{W}, \omega_{\bar{W}}\right)
$$

is a symplectic embedding.

Fix a neighbourhood $U(L)$ of $L$ with $\overline{U(L)} \subset V_{\phi<c}$.

Denote by $\mathcal{J}^{\infty}$ the space of two-parametric families of smooth almost complex structures $\mathbf{J}=\left\{J_{s, t}\right\}_{(s, t) \in \mathbb{R} \times[0,1]}$ on $\bar{W}$ that have the following properties:

1. $J_{s, t}$ is an almost complex structure compatible with $\omega_{\bar{W}}$ for every $s, t$. 
2. $J_{s, t}$ does not depend on $(s, t)$ for $|s|$ large enough or when $t=0,1$.

3. $J_{s, t} \equiv J_{\bar{W}}$ on $\bar{W} \backslash U(L)$ for every $s, t$.

In what follows we shall call an element $\mathbf{J} \in \mathcal{J}^{\infty}$ autonomous if it does not depend on $(s, t)$.

Fix a family $\left\{\rho_{R}\right\}_{0 \leq R<\infty}$ of smooth cut-off functions $\rho_{R}: \mathbb{R} \rightarrow[0,1]$ that depends smoothly on the parameter $R \in[0, \infty)$ and such that

1. $\rho_{R} \equiv 1$ on $[-R, R]$ and $\operatorname{supp}\left(\rho_{R}\right) \subset[-R-1, R+1]$ for every $R \geq 1$.

2. $\rho_{R}^{\prime}<0$ on $(R, R+1)$ and $\rho_{R}^{\prime}>0$ on $(-R-1,-R)$ for every $R \geq 1$.

3. $\rho_{R}=R \rho_{1}$ for every $0 \leq R \leq 1$.

Let $H: \bar{W} \times[0,1] \rightarrow \mathbb{R}$ be a given compactly supported Hamiltonian function on $\bar{W}$. For every $\mathbf{J} \in \mathcal{J}^{\infty}$ and $R \geq 0$ consider smooth maps $u: \mathbb{R} \times[0,1] \rightarrow \bar{W}$ that satisfy the following perturbed Cauchy-Riemann equation:

$$
\begin{cases}\partial_{s} u+J_{s, t}(u) \partial_{t} u=\rho_{R}(s) J_{s, t}(u) \xi_{H}(t, u) & s \in \mathbb{R}, t \in[0,1] \\ u(s, 0), u(s, 1) \in L & \text { for every } s \in \mathbb{R}\end{cases}
$$

and have finite energy

$$
E_{\mathbf{J}}(u)=\int_{\mathbb{R} \times[0,1]}\left\|\partial_{t} u\right\|_{g_{s_{s, t}}}^{2} d s d t<\infty
$$

Here $\xi_{H}$ stands for the Hamiltonian vector field corresponding to the function $H$. Using a conformal change of coordinates $(\mathbb{R} \times[0,1], \mathbb{R} \times\{0,1\}) \cong$ $\left(D^{2} \backslash\{-1,1\}, \partial D^{2} \backslash\{-1,1\}\right)$ we get from each solution $u$ of the above problem a finite energy map $\left(D^{2} \backslash\{-1,1\}, \partial D^{2} \backslash\{-1,1\}\right) \rightarrow(\bar{W}, L)$ which is $J$ holomorphic near $-1,1 \in D^{2}$ for some autonomous almost complex structure $J$. By the removal of singularity theorem of $\mathrm{Oh}[\mathrm{Oh}-1]$ this map extends to a smooth map, which we still denote by $u:\left(D^{2}, \partial D^{2}\right) \rightarrow(\bar{W}, L)$. We write $[u] \in \pi_{2}(\bar{W}, L)$ for the homotopy class of this map.

Let $\mathcal{J} \subset \mathcal{J}^{\infty}$ be a Banach space of smooth perturbations of $J_{\bar{W}}$ as defined in $[\mathrm{F}-1]$. Let $W^{1, p}(\mathbb{R} \times[0,1], \bar{W})(p>2)$ be the Banach manifold of Sobolev $(1, p)$ maps as defined in [Sc] (i.e., with a nonstandard measure on $\mathbb{R}$ ).

For $A \in \pi_{2}(\bar{W}, L)$ and $R \in \mathbb{R}$ denote by

$$
\mathcal{M}_{H, R}(A ; \mathcal{J}) \subset W^{k, p}(\mathbb{R} \times[0,1], \bar{W}) \times \mathcal{J}
$$

the space of all pairs $(u, \mathbf{J})$ for which $u$ is a finite energy solution of equation (1) (with parameter $R$ ), and $[u]=A \in \pi_{2}(\bar{W}, L)$. Finally, write $P_{A, R}: \mathcal{M}_{H, R}(A ; \mathcal{J}) \rightarrow \mathcal{J}$ for the natural projection.

The following are all standard facts: 
1. The space $\mathcal{M}_{H, R}(A ; \mathcal{J})$ does not depend on $p>2$, and every $u \in \mathcal{M}_{H, R}(A ; \mathcal{J})$ is smooth.

2. $\mathcal{M}_{H, R}(A ; \mathcal{J})$ is a smooth Banach manifold, and the projection $P_{A, R}$ is Fredholm of index $\mu(A)+\operatorname{dim} L$.

3. For every $R \in \mathbb{R}$ the subset $\mathcal{J}_{\text {reg, } R} \subset \mathcal{J}$ consisting of all J's that are regular values of $P_{A, R}$ for every $A \in \pi_{2}(\bar{W}, L)$ is of second category. Note that this implies that there exists a second category subset $\mathcal{J}_{\text {reg }} \subset \mathcal{J}$ and a dense subset $\mathcal{R} \subset \mathbb{R}$ such that for every $\mathbf{J} \in \mathcal{J}$ and $R \in \mathcal{R}$ we have $\mathbf{J} \in \mathcal{J}_{\text {reg, } R}$.

4. In particular, for every $\mathbf{J} \in \mathcal{J}_{\mathrm{reg}, R}$ the space $\mathcal{M}_{H_{3} R}(A ; \mathbf{J})=P_{A, R}^{-1}(\mathbf{J})$ of finite energy solutions of (1) is either empty or a smooth manifold of dimension $\mu(A)+\operatorname{dim} L$.

5. Given two pairs $\left(\mathbf{J}_{0}, R_{0}\right),\left(\mathbf{J}_{1}, R_{1}\right)$ such that $\mathbf{J}_{0} \in \mathcal{J}_{\text {reg, } R_{0}}$ and $\mathbf{J}_{1} \in \mathcal{J}_{\text {reg, }, R_{1}}$ there exists a second category subset $\mathcal{P}_{\text {reg }}$ in the space of smooth paths $\left\{\left(\mathbf{J}_{\lambda}, R_{\lambda}\right)\right\}_{0 \leq \lambda \leq 1}$ that connect $\left(\mathbf{J}_{0}, R_{0}\right)$ with $\left(\mathbf{J}_{1}, R_{1}\right)$ such that for every $\left\{\left(\mathbf{J}_{\lambda}, R_{\lambda}\right)\right\}_{0 \leq \lambda \leq 1} \in \mathcal{P}_{\text {reg }}$ the space

$$
\mathcal{M}_{H}\left(A ;\left\{\left(\mathbf{J}_{\lambda}, R_{\lambda}\right)\right\}\right)=\left\{\left(u, \mathbf{J}_{\lambda}, R_{\lambda}, \lambda\right) \mid u \in \mathcal{M}_{H, R_{\lambda}}\left(A ; \mathbf{J}_{\lambda}\right) \forall 0 \leq \lambda \leq 1\right\}
$$

is either empty or a smooth manifold of dimension $\mu(A)+\operatorname{dim} L+1$ with boundary consisting of the disjoint union

$$
\mathcal{M}_{H, R_{0}}\left(A ; \mathbf{J}_{0}\right) \times\left\{\left(R_{0}, 0\right)\right\} \coprod \mathcal{M}_{H, R_{1}}\left(A ; \mathbf{J}_{1}\right) \times\left\{\left(R_{1}, 1\right)\right\}
$$

Proposition 4.1: Let $H: \bar{W} \times[0,1] \rightarrow \mathbb{R}$ be a compactly supported Hamiltonian function. Then there exists a compact subset $K \subset V$ such that for every $\mathbf{J} \in \mathcal{J}$ and $R \in \mathbb{R}$ all finite energy solutions $u$ of equation (1) must lie entirely inside $K \times X$.

Proof: Put

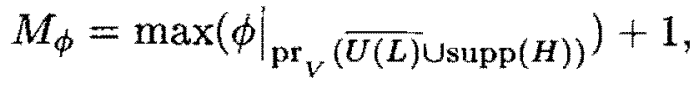

and take $K$ to be the compact subset $V_{\phi \leq M_{\phi}}$.

Suppose that $u$ is a solution of (1) that goes outside of $K \times X$. From the definition of the space $\mathcal{J}$ it follows that $u$ must be $J_{\bar{W}}$-holomorphic at points that go outside $K \times X$. Note that the image of $u$ cannot be entirely outside $K \times X$ because $u$ sends $\mathbb{R} \times\{0,1\}$ to $L \subset K \times X$. Thus, there exists a disk $D_{0} \subset \operatorname{Int}(\mathbb{R} \times[0,1])$ such that $\left.u\right|_{D_{0}}$ is $J_{\bar{W}}$-holomorphic, $u\left(\partial D_{0}\right) \subset K \times X$, but $u\left(\right.$ Int $\left.D_{0}\right)$ is not contained in $K \times X$. The projection $\operatorname{pr}_{v} \circ u\left(D_{0}\right)$ is therefore a $J_{V}$-holomorphic disk on which the restriction of $\phi$ has a maximum at an interior 
point. By the maximum principle $\phi$ must be constant on $\operatorname{pr}_{v} \circ u\left(D_{0}\right)$ which is a contradiction because $\mathrm{pr}_{V} \circ u\left(\partial D_{0}\right)$ lies in $K$.

The following proposition is also standard and can be proved using similar methods to $[\mathrm{F}-1, \mathrm{Oh}-3, \mathrm{P}]$ :

Proposition 4.2:

1. (cf. [Oh-3]). Every autonomous $\mathrm{J} \in \mathcal{J}$ is a regular value of $P_{0,0}$. In this case, every $u \in \mathcal{M}_{H, 0}(0 ; \mathbf{J})$ is constant, hence $\mathcal{M}_{H, 0}(0 ; J)$ is naturally diffeomorphic to $L$, and in particular it is compact.

2. (cf. [P]). For every autonomous $\mathbf{J} \in \mathcal{J}$ there exists neighbourhood $\mathcal{B}_{\mathbf{J}}$ of $(\mathbf{J}, 0)$ in $\mathcal{J} \times \mathbb{R}$ so that the projection

$$
\left\{\left(u, \mathbf{J}^{\prime}, R\right) \mid u \in \mathcal{M}_{H, R}\left(0 ; \mathbf{J}^{\prime}\right)\right\} \ni\left(u, \mathbf{J}^{\prime}, R\right) \longmapsto\left(\mathbf{J}^{\prime}, R\right) \in \mathcal{B}_{\mathbf{J}}
$$

is proper. In particular, for every $\left(\mathbf{J}^{\prime}, R\right) \in \mathcal{B}_{\mathbf{J}}$ the space $\mathcal{M}_{H, R}\left(0 ; \mathbf{J}^{\prime}\right)$ is compact; moreover it is not empty.

The next proposition, due to $\mathrm{Oh}$ (see also Chekanov [Ch]), will play a crucial role in the rest of the proof.

Proposition 4.3 (cf. [Oh-4]): Suppose that the time-1 map $F: \bar{W} \rightarrow \bar{W}$ of the flow generated by the (compactly supported) Hamiltonian $H: \bar{W} \times[0,1] \rightarrow \mathbb{R}$ satisfies $F(L) \cap L=\emptyset$. Then for every $\mathbf{J} \in \mathcal{J}$ there exists $R_{1}>0$ such that for every $R \geq R_{1}$ equation (1) does not admit any finite energy solutions in the class $0 \in \pi_{2}(\bar{W}, L)$, that is $\mathcal{M}_{H, R}(0 ; \mathbf{J})=\emptyset$.

We now bring the subcriticality of $V$ into the play. By Lemma 3.2, there exists a compactly supported Hamiltonian diffeomorphism $f:\left(V, \omega_{\phi}\right) \rightarrow\left(V, \omega_{\phi}\right)$ such that

$$
f\left(\operatorname{pr}_{V}(L)\right) \cap \operatorname{pr}_{V}(L)=\emptyset .
$$

Let $H_{V}: V \times[0,1] \rightarrow \mathbb{R}$ be a compactly supported Hamiltonian function that generates $f$ as its time-1 map. Pick two neighbourhoods $U^{\prime \prime} \supset U^{\prime} \supset \operatorname{pr}_{x}(L)$ of $\operatorname{pr}_{X}(L)$ with compact closure in $X$ and such that $U^{\prime \prime} \supset \overline{U^{\prime}}$. Consider now a Hamiltonian function $H=\bar{W} \times[0,1] \rightarrow \mathbb{R}$ that has the following properties:

- $\operatorname{supp}(H) \subset \operatorname{supp}\left(H_{V}\right) \times U^{\prime \prime}$.

- $H(v, x, t)=H_{V}(v, t)$ on $V \times U^{\prime}$.

Finally, denote by $F$ the time-1 map of $H$. Clearly $F$ coincides with $f \times$ Id on $V \times U^{\prime}$ and since $L \subset V \times U^{\prime}$ we have $F(L) \cap L=\emptyset$.

Let $\mathbf{J}$ be any autonomous almost complex structure in $\mathcal{J}$. Choose a pair $\left(\mathbf{J}_{0}, R_{0}\right) \in \mathcal{B}_{\mathbf{J}}$ such that $\mathbf{J}_{0} \in \mathcal{J}_{\text {reg }}$ and $R_{0} \in \mathcal{R}$. Next, fix a pair $\left(\mathbf{J}_{1}, R_{1}\right)$ with 
$\mathbf{J}_{1} \in \mathcal{J}_{\text {reg }}$ and $0<R_{1} \in \mathcal{R}$ large enough, according to Proposition 4.3, so that $\mathcal{M}_{H, R_{1}}\left(A ; \mathbf{J}_{1}\right)$ is empty. Due to these choices we can connect the pairs $\left(\mathbf{J}_{0}, R_{0}\right)$, $\left(\mathrm{J}_{1}, R_{1}\right)$ by a path $\left\{\left(\mathrm{J}_{\lambda}, R_{\lambda}\right)\right\}_{0 \leq \lambda \leq 1}$ belonging to $\mathcal{P}_{\text {reg }}$.

Our choice of the initial pair $\left(\mathbf{J}_{0}, R_{0}\right)$ and the fact that $\mathcal{M}_{H, R_{1}}\left(0 ; \mathbf{J}_{1}\right)$ is empty imply that the manifold $\mathcal{M}_{H}\left(0 ;\left\{\left(\mathbf{J}_{\lambda}, R_{\lambda}\right)\right\}\right)$ cannot be compact (see [Oh-3]). We now apply Gromov's compactness theorem [Gr]. In view of Proposition 4.1, Gromov's compactness theorem still applies in our setting although the manifold $\bar{W}$ is not compact.

It now follows from Gromov's compactness theorem that there exists $0 \leq \lambda_{*} \leq$ 1 for which bubbling must occur. Again, due to our choice of $\left(\mathrm{J}_{0}, R_{0}\right)$ this $\lambda_{*}$ must be strictly positive. Thus there exist a sequence $\lambda_{n}$ with $\lambda_{n} \rightarrow \lambda_{*}$ and a sequence $\left(u_{n}, R_{\lambda_{n}}\right)$ with $u_{n} \in \mathcal{M}_{H, R_{\lambda_{n}}}\left(0 ; \mathbf{J}_{\lambda_{n}}\right)$ such that $u_{n}$ weakly converges to a cusp curve

$$
u_{\infty}=v_{0}+\sum_{i=1}^{p} v_{i}^{\prime}+\sum_{j=1}^{q} v_{j}^{\prime \prime} \quad(p+q \geq 1, p, q \geq 0),
$$

where:

1. $v_{0}:(\mathbb{R} \times[0,1], \mathbb{R} \times\{0,1\}) \rightarrow(\bar{W}, L)$ is a finite energy solution of (1) for the pair $\left(\mathbf{J}_{\lambda_{*}}, R_{\lambda_{*}}\right)$. To describe the other components write $\mathbf{J}_{\lambda_{*}}=$ $\left\{J_{s, t}\right\}_{(s, t) \in \mathbb{R} \times[0,1]}$.

2. For every $1 \leq i \leq p, v_{i}^{\prime}:\left(D^{2}, \partial D^{2}\right) \rightarrow(\widetilde{W}, L)$ is a nonconstant $J_{s, 0^{-}}$ holomorphic disk (recall that $J_{s, 0}=J_{s, 1}$ does not depend on $s$ ).

3. For every $1 \leq j \leq q, v_{j}^{\prime \prime}: \mathbb{C} P^{1} \rightarrow \bar{W}$ is a nonconstant $J_{s_{j}, t_{j}}$-holomorphic sphere for some $\left(s_{j}, t_{j}\right) \in \mathbb{R} \times[0,1]$.

Note that it is possible that we have only disks or only spheres in the sum (2); however, at least one of these two possibilities must occur. Moreover, the total sum of homotopy classes in $\pi_{2}(\bar{W}, L)$ is preserved, namely:

$$
0=\left[u_{\lambda_{n}}\right]=\left[u_{\infty}\right]=\left[v_{0}\right]+\sum_{i=1}^{p}\left[v_{i}^{\prime}\right]+\sum_{j=1}^{q} i_{*}\left[v_{j}^{\prime \prime}\right] .
$$

Here, $\left[u_{\lambda_{n}}\right],\left[u_{\infty}\right],\left[v_{0}\right],\left[v_{i}^{\prime}\right] \in \pi_{2}(\bar{W}, L)$ stand for the homotopy classes of the corresponding disks, $\left[v_{j}^{\prime \prime}\right] \in \pi_{2}(\bar{W})$ for the homotopy classes of the corresponding spheres and $i_{*}: \pi_{2}(\bar{W}) \rightarrow \pi_{2}(\bar{W}, L)$ for the obvious homomorphism.

Put $A_{0}=\left[v_{0}\right] \in \pi_{2}(\bar{W}, L)$. By our choice of the path $\left\{\left(\mathbf{J}_{\lambda}, R_{\lambda}\right)\right\}_{0 \leq \lambda \leq 1}$ the space $\mathcal{M}_{H}\left(A_{0} ;\left\{\left(\mathbf{J}_{\lambda}, R_{\lambda}\right)\right\}\right)$ is either empty or a smooth manifold of dimension $\mu\left(A_{0}\right)+\operatorname{dim} L+1$. But clearly this space is not empty because $\left(v_{0}, \mathbf{J}_{\lambda_{*}}, R_{\lambda_{*}}, \lambda_{*}\right)$ lies in it. We thus have

$$
\mu\left(A_{0}\right)+\operatorname{dim} L+1 \geq 0 .
$$


Applying $\mu$ to the sum in (3) we obtain

$$
\sum_{i=1}^{p} \mu\left(\left[v_{i}^{\prime}\right]\right)+2 \sum_{j=1}^{q} c_{1}^{\bar{W}}\left(\left[v_{j}^{\prime \prime}\right]\right) \leq 1+\operatorname{dim} L
$$

Now choose a sequence $\left\{\left(\mathbf{J}_{\lambda}^{(n)}, R_{\lambda}^{(n)}\right)\right\}_{0 \leq \lambda \leq 1}$ of regular paths as above converging to a path $\left\{\left(\mathbf{J}_{\lambda}^{\infty}, R_{\lambda}^{\infty}\right)\right\}_{0 \leq \lambda \leq 1}$ such that $\mathbf{J}_{\lambda}^{\infty}=\left\{J_{\bar{W}}\right\}$ is the constant path given by the autonomous complex structure $J_{\bar{W}}$. Here we can achieve convergence of the paths $R_{\lambda}^{(n)}$ because, by the same indirect proof as in [Oh-4], the constant $R_{1}$ in Proposition 4.3 can be chosen uniformly for a neighbourhood of $\left\{J_{\bar{W}}\right\}$.

The corresponding sequence of cusp curves $\left(v_{i}^{\prime(n)}, v_{j}^{\prime \prime(n)}\right)$ has uniformly bounded area. So again by Gromov compactness, it will have a subsequence that converges to a $J_{\bar{W}}$-holomorphic cusp curve $\left(v_{1}^{\prime}, \ldots, v_{p}^{\prime}, v_{1}^{\prime \prime}, \ldots, v_{q}^{\prime \prime}\right)$. Here the number of disks and spheres in the sequence of cusp curves may increase, but it remains finite because the areas of the disks and spheres are uniformly bounded from below. Moreover, the inequality (4) still holds in the limit.

Consequently, either there exists $1 \leq i_{0} \leq p$ with $\mu\left(\left[v_{i_{0}}^{t}\right]\right) \leq 1+\operatorname{dim} L$, or there exists $1 \leq j_{0} \leq q$ with $2 c_{1}^{\bar{W}}\left(\left[v_{j 0}^{\prime \prime}\right]\right) \leq 1+\operatorname{dim} L$. If the first possibility occurs denote by $D$ the $J_{\bar{W}}$-holomorphic disk $v_{i_{0}}^{\prime}$ and in the second case write $S$ for the $J_{\bar{W}}$-holomorphic sphere $v_{j_{0}}^{\prime \prime}$. By Proposition 4.1 we have that in both cases $D$ and $S$ must lie entirely inside $V_{\varphi<c} \times X$.

Note that either of $D$ and $S$ might not be reduced. Let us deal first with the case of a sphere $S$. If $S$ is not reduced then it is well known that it must be multiply covered. Let $S_{\text {red }}$ be the reduction of $S$. Then $[S]$ is just a positive multiple of $\left[S_{\text {red }}\right]$, so if $2 c_{1}^{\bar{W}}([S]) \leq \operatorname{dim} L+1$ then the same inequality will hold also for $\left[S_{\text {red }}\right]$. In other words, replacing $S$ by its reduction will not destroy the upper bound on the first Chern number.

The case when $D$ is not reduced is much more complicated since such a disk might not be multiply covered (see $[\mathrm{K}-\mathrm{O}, \mathrm{La}]$ for examples of this phenomenon). However, using the techniques of either Kwon and Oh [K-O] or Lazzarini [La] it is possible to decompose the disk $D$ into a sum of multiply covered $J_{\bar{W}}$-holomorphic disks, namely there exist reduced $J_{\bar{W}}$-holomorphic disks $D_{1}, \ldots, D_{r}$ with boundary on $L$ and $m_{1}, \ldots, m_{r} \in \mathbb{N}$ such that:

1. The union of images of the $D_{i}$ 's equal to the image of $D$.

2. $[D]=\sum_{i=1}^{r} m_{i}\left[D_{i}\right]$ in $H_{2}(\bar{W}, L)$.

Since $\mu[D] \leq \operatorname{dim} L+1$ there exists $1 \leq i_{0} \leq r$ such that $\mu\left[D_{i_{0}}\right] \leq \operatorname{dim} L+1$.

Summarizing the above, replacing $S$ by $S_{\text {red }}$ or $D$ by $D_{i_{0}}$ we may assume from now on that both of $S$ and $D$ are reduced. 
It remains to prove the lower bound $\mu([D]) \geq 3-\operatorname{dim} L$ in case we have a disk $D$. For this purpose denote by $\mathcal{J}_{*} \subset \mathcal{J}$ the space of all autonomous almost complex structures $\mathbf{J}=\left\{J_{s, t} \equiv J\right\}$. Note that our definition of the space $\mathcal{J}$ allows us to vary $J$ arbitrarily inside $U(L)$ (as long as it remains compatible with $\omega_{\bar{W}}$ ), and every nonconstant $J$-holomorphic disk with boundary on $L$ must pass through $U(L)$. A standard transversality argument shows that there exists a second category subset $\mathcal{J}_{* \text {,reg }} \subset \mathcal{J}_{*}$ such that for every $J \in \mathcal{J}_{* \text {,reg }}$ and $B \in \pi_{2}(W, L)$ the space $\mathcal{M}_{J}(B)$ of non-parametrized somewhere injective $J$-holomorphic disks $v:\left(D^{2}, \partial D^{2}\right) \rightarrow(W, L)$ that represent the class $B$ is either empty or a smooth manifold of dimension $\mu(B)+\operatorname{dim} L-3$. Fix such a $J \in \mathcal{J}_{* \text {,reg }}$.

Now repeat the argument above with $J_{\bar{W}}$ replaced by $J$. We obtain either a reduced $J$-holomorphic sphere $S$ or a reduced $J$-holomorphic disk $D$ with $\mu([D]) \leq \operatorname{dim} L+1$. Assume we obtain a disk $D$. Since the disk $D$ is reduced it follows from our choice of $J$ that $\mathcal{M}_{J}([D])$ is a non-empty manifold. Therefore $\mu([D])+\operatorname{dim} L-3 \geq 0$.

Proof of Theorem 2.2: Let $U_{0}(L)$ be a Weinstein neighbourhood of $L$ with $\overline{U_{0}(L)} \subset U(L)$. Consider the completed manifold $\left(\bar{W}, \omega_{\bar{W}}\right)$ as in the proof of Theorem 2.1. By Lemma 3.2, $L$ can be disjoined from itself by the time-1 map of a compactly supported Hamiltonian $H$.

Consider the "local" Floer cohomology $H F_{\text {loc }}^{*}\left(L, L ; U_{0}(L) ; \mathbb{Z}_{2}\right)$ and the "global" Floer cohomology $H F\left(L, L ; \bar{W} ; \mathbb{Z}_{2}\right)$ as defined in $[\mathrm{Oh}-3]$. Here for the global Floer cohomology we allow only deformations generated by Hamiltonians whose Hofer norm is at most the Hofer norm of $H$. It is proved in [Oh-2] that there exists a constant $c$, depending only on the Hofer norm of $H$, such that all gradient trajectories in the Floer complex have energy uniformly bounded by $c$.

Now assume that there exists a regular almost complex structure $J$ that agrees with $J_{\bar{W}}$ outside $U(L)$ and such that

1. For every nonconstant $J$-holomorphic disk $(D, \partial D) \subset(\bar{W}, L)$ of area $\leq c$, $\mu([D]) \geq \operatorname{dim} L+1$.

2. For every nonconstant $J$-holomorphic sphere $S \subset \bar{W}$ of area $\leq c, 2 c_{1}([S]) \geq$ $\operatorname{dim} L+1$.

Under this assumption the global Floer cohomology is well defined and invariant under (compactly supported) Hamiltonian deformations because $\operatorname{dim} L+1 \geq 3$ (see [Oh-2,Oh-3]). Moreover it follows from [Oh-3] that under the above assumption the global Floer cohomology is isomorphic to the local Floer cohomology except maybe for dimensions $k=0, \operatorname{dim} L$. More precisely, $H F\left(L, L ; \bar{W} ; \mathbb{Z}_{2}\right)$ is 
isomorphic either to

$$
\bigoplus_{k=0}^{\operatorname{dim} L} H F_{\mathrm{loc}}^{k}\left(L, L ; U_{0}(L) ; \mathbb{Z}_{2}\right), \quad \text { or to } \bigoplus_{k=1}^{\operatorname{dim} L-1} H F_{\mathrm{loc}}^{k}\left(L, L ; U_{0}(L) ; \mathbb{Z}_{2}\right)
$$

Let us briefly outline the main ideas behind this (precise details can be found in Sections 4 and 5 of [Oh-3]). Recall that the local Floer cohomology is defined as follows: pick a $C^{2}$-small Morse function $G$ on $L$ and let $L_{G} \subset U_{0}(L)$ be the Hamiltonian image of $L$ obtained using $G$ (i.e., $L_{G}$ is the graph of the 1-form $d G$ in $U_{0}(L)$ viewed as a neighbourhood of the zero section in $\left.T^{*} L\right)$. The Floer complex is generated by the points of $L \cap L_{G}$ which are precisely the critical points of $G$. The differential for the local Floer homology however takes into account only trajectories that lie entirely inside $U_{0}(L)$. The resulting cohomology, $H F_{\text {loc }}^{*}\left(L, L ; U_{0}(L) ; \mathbb{Z}_{2}\right)$ (is independent of $G$ and) is called the local Floer cohomology. If, the first possibility described in (5) does not hold then for any choice of $G$ as above there must be a Floer trajectory $u$ of (relative) index 1 connecting two critical points $x, y \in \operatorname{Crit}(G)$, but such that $u$ goes outside of $U_{0}(L)$. If in addition, the second possibility of (5) does not hold either, then there exist $x, y$ as above with

$$
1 \leq \operatorname{ind}_{y} G-\operatorname{ind}_{x} G \leq \operatorname{dim} L-1
$$

Repeating this argument for $\frac{1}{n} G$ and $L_{\frac{1}{n} G}$ we get a sequence of Floer trajectories $u_{n}$ of relative index 1 connecting two points $x_{n}, y_{n} \in \operatorname{Crit}\left(\frac{1}{n} G\right)=\operatorname{Crit}(G)$ that satisfy $(6)$ and such that none of the $u_{n}$ 's is entirely contained inside $U_{0}(L)$. Passing to a subsequence if necessary we obtain in the limit as $n \rightarrow \infty$ a cusp trajectory $u_{\infty}$ connecting two critical points $x^{\prime}, y^{\prime} \in \operatorname{Crit}(G)$ satisfying (6). This cusp trajectory consists of possibly some $J$-holomorphic discs $D_{i}$ with boundary on $L$, some $J$-holomorphic spheres $S_{j}$ and some gradient trajectories lying on $L$. Since none of the $u_{n}$ 's was contained inside $U_{0}(L)$ the same holds also for $u_{\infty}$. Therefore $u_{\infty}$ must contain at least one $J$-holomorphic disc or sphere. An index computation (see [Oh-3] Section 5) gives:

$$
0<\sum \mu\left(\left[D_{i}\right]\right)+\sum 2 c_{1}\left(\left[S_{j}\right]\right)=1-\left(\operatorname{ind}_{x^{\prime}} G-\operatorname{ind}_{y^{\prime}} G\right) \leq \operatorname{dim} L
$$

where the leftmost inequality follows from monotonicity, while the rightmost one is due to the fact that $x^{\prime}, y^{\prime}$ satisfy (6). Let $D$ be one of the discs or spheres appearing in $u_{\infty}$. Since we are in the monotone situation we conclude that $\mu([D]) \leq \operatorname{dim} L$. This gives a contradiction to our assumptions 1,2 above. Thus 
one of the possibilities described in (5) holds. We refer the reader to [Oh-3, Oh-5] for more details on the isomorphisms (5).

Let us return now to the present situation. Since $L$ is disjoined from itself by the time-1 map of $H, H F\left(L, L ; \bar{W} ; \mathbb{Z}_{2}\right)=0$. On the other hand, by $[\mathrm{Oh}-3]$ we know that $H F_{\text {loc }}^{*}\left(L, L ; U_{0}(L) ; \mathbb{Z}_{2}\right) \cong H^{*}\left(L ; \mathbb{Z}_{2}\right)$. Therefore, by $(5)$ we infer that $H^{k}\left(L ; \mathbb{Z}_{2}\right)=0$ for every $1 \leq k \leq \operatorname{dim} L-1$ (note that by assumption $\operatorname{dim} L \geq 2$ ).

If the assumptions 1 and 2 do not hold, then there exists a sequence of almost complex structures $J_{n}$ converging to $J_{\bar{W}}$ and nonconstant $J_{n}$-holomorphic curves (disks or spheres) $u_{n}$ of area $\leq c$ such that $\mu\left(\left[u_{n}\right]\right) \leq \operatorname{dim} L$. By Gromov compactness, $u_{n}$ converges to a cusp curve that contains a nonconstant $J_{\bar{W}}$-holomorphic disk or sphere of Maslov index $\leq \operatorname{dim} L$.

Proof of Theorem 2.5: By the definition of displacement energy, since $L$ is contained in the interior of $V \times X$, we can choose the Hamiltonian $H$ in the proof of Theorem 2.1 that displaces $L$ from itself to satisfy

$$
\|H\| \leq e\left(V, J_{V}, \phi\right)
$$

It is shown in [Oh-4] that the energy of every solution $u$ of equation (1) is bounded by

$$
E_{\mathbf{J}}(u) \leq e\left(V, J_{V}, \phi\right)
$$

Therefore, this energy estimate holds for every component of the cusp curves.

Proof of Theorem 2.8: Let $\Sigma \subset X$ be a properly embedded $J_{X}$-complex hypersurface which is spherically nef. Choose the neighbourhood $U(L)$ from the beginning of the proof of Theorem 2.1 small enough so that its closure is disjoint from $V \times \Sigma$. Let $u_{\infty}=v_{0}+\sum_{i=1}^{p} v_{i}^{\prime}+\sum_{j=1}^{q} v_{j}^{\prime \prime}$ be a cusp curve as in equation (2) in the proof of Theorem 2.1. We claim that for every $1 \leq i \leq p$ and $1 \leq j \leq q$ we have:

1. $[V \times \Sigma] \cdot\left[v_{i}^{\prime}\right] \geq 0$ with equality if and only if the image of $v_{i}^{\prime}$ is disjoint from $V \times \Sigma$.

2. $[V \times \Sigma] \cdot\left[v_{j}^{\prime \prime}\right] \geq 0$ with equality only if either $v_{j}^{\prime \prime}$ is disjoint from $V \times \Sigma$ or is completely contained inside $V \times \Sigma$ in which case it must be $J_{W}$-holomorphic.

3. $[V \times \Sigma] \cdot\left[v_{0}\right] \geq 0$ with equality if and only if the image of $v_{0}$ is disjoint from $V \times \Sigma$.

Indeed, by our definition of the space $\mathcal{J}$ every $\mathbf{J} \in \mathcal{J}$ coincides with the autonomous structure $J_{\bar{W}}=J_{V} \oplus J_{X}$ outside of $U(L)$. Therefore, all of 
$v_{1}^{\prime}, \ldots, v_{p}^{\prime}, v_{1}^{\prime \prime}, \ldots, v_{q}^{\prime \prime}$ must be $J_{\bar{W}}$-holomorphic at points that go through the complement of $U(L)$. As $V \times \Sigma$ is $J_{W^{-}}$-holomorphic too, any intersection between $V \times \Sigma$ and $v_{i}^{\prime}$ must contribute positively to the total intersection $[V \times \Sigma] \cdot\left[v_{i}^{\prime}\right]$, unless $v_{i}^{\prime}$ is totally contained inside $V \times \Sigma$. But clearly, none of the disks $v_{i}^{\prime}$ is contained in $V \times \Sigma$, because their boundaries are on $L$. This proves 1. A similar argument shows that if $v_{j}^{\prime \prime}$ is not contained inside $V \times \Sigma$ then $[V \times \Sigma] \cdot\left[v_{j}^{\prime \prime}\right] \geq 0$ with equality if and only if $v_{j}^{\prime \prime}$ is in the complement of $V \times \Sigma$. In case $v_{j}^{\prime \prime}$ is contained inside $V \times \Sigma$ it is $J_{W}$-holomorphic and the assumption that $\Sigma$ is spherically nef assures that we still have $[V \times \Sigma] \cdot\left[v_{j}^{\prime \prime}\right] \geq 0$. This proves 2 .

For statement 3 , recall that Hamiltonian function $H$ that we use in the proof of Theorem 2.1 to disjoin $L$ from itself had support inside $V \times U^{\prime \prime}$, where $U^{\prime \prime}$ could be taken as an arbitrary neighbourhood of $\operatorname{pr}_{X}(L)$. Since the hypersurface $\Sigma$ is disjoint from $\operatorname{pr}_{X}(L)$ we can choose $U^{\prime \prime}$ to be disjoint from $\Sigma$. Thus we may assume that the Hamiltonian vector field $\xi_{H}(t, \cdot)$ vanishes in a neighbourhood of $V \times \Sigma$. Since $v_{0}$ is a solution to equations (1), this implies that it is $J_{\bar{W}}$ holomorphic near $V \times \Sigma$. Therefore $v_{0}$ must intersect $V \times \Sigma$ non-negatively unless $\operatorname{pr}_{X} \circ v_{0}$ is entirely contained inside of $\Sigma$. The latter possibility however cannot occur because the boundary of $v_{0}$ lies in $L$ and $\operatorname{pr}_{x}(L)$ is disjoint from $\Sigma$ by assumption. Finally, if we have $[V \times \Sigma] \cdot\left[v_{0}\right]=0$ then as before, since each intersection point between $v_{0}$ and $V \times \Sigma$ contributes positively to the total intersection, the image of $v_{0}$ must be disjoint from $V \times \Sigma$. This proves 3 .

Summing up all the above we see that all components of the cusp curve $u_{\infty}$ intersect $V \times \Sigma$ non-negatively. On the other hand, from equation (3) we get that the total sum of these homological intersections is zero. Therefore the homological intersection of each component of our cusp curve with $V \times \Sigma$ is zero.

Now, as in the proof of Theorem 2.1, we take a sequence of regular paths converging to the constant path $\left\{J_{\bar{W}}\right\}$. Since the homology classes converge, the resulting $J_{\bar{W}}$-holomorphic disks and spheres also have homological intersection zero with $V \times \Sigma$. By what we have just proved this implies that each of the disks $v_{i}^{\prime}$ lies in the complement of $V \times \Sigma$ and each of the spheres $v_{j}^{\prime \prime}$ is either in the complement of $V \times \Sigma$ or entirely contained inside $V \times \Sigma$. Finally, recall that cusp curves must be connected, hence it is impossible to have some of the spheres above entirely contained inside $V \times \Sigma$ while all the other components are disjoint from $V \times \Sigma$. Thus all the components of our cusp curve are in the complement of $V \times \Sigma$. 


\section{References}

[A] M. Audin, Fibrés normaux d'immersions en dimension double, points doubles d'immersions lagragiennes et plongements totalement réels, Commentarii Mathematici Helvetici 63 (1988), 593-623.

[A-L-P] M. Audin, F. Lalonde and L. Polterovich, Symplectic rigidity: Lagrangian submanifolds, in Holomorphic Curves in Symplectic Geometry (M. Audin and J. Lafontaine, eds.), Progress in Mathematics, 117, Birkhäuser Verlag, Basel, 1994.

[Bi] P. Biran, Lagrangian barriers and symplectic embeddings, to appear in Geometric and Functional Analysis.

[B-C] P. Biran and K. Cieliebak, Symplectic topology on subcritical manifolds, to appear in Commentarii Mathematici Helvetici.

[Ch] Yu. V. Chekanov, Lagrangian intersections, symplectic energy, and areas of holomorphic curves, Duke Mathematical Journal 95 (1998), $213-226$.

[E] Y. Eliashberg, Topological characterization of Stein manifolds of dimension $>2$, International Journal of Mathematics 1 (1990), no. 1, 29-46.

[E-G] Y. Eliashberg and M. Gromov, Convex symplectic manifolds, in Several Complex Variables and Complex Geometry, Part 2 (Santa Cruz, CA, 1989), Proceedings of the Symposium Pure Mathematics, 52, Part 2, American Mathematical Society, Providence, RI, 1991, pp. 135-162.

[F-1] A. Floer, The unregularized gradient flow of the symplectic action, Communications on Pure and Applied Mathematics 41 (1988), 775-813.

[F-2] A. Floer, Morse theory for Lagrangian intersections, Journal of Differential Geometry 28 (1988), 513-547.

[Gr] M. Gromov, Pseudoholomorphic curves in symplectic manifolds, Inventiones Mathematicae 82 (1985), 307-347.

[H-Z] H. Hofer and E. Zehnder, Symplectic Invariants and Hamiltonian Dynamics, Birkhäuser Verlag, Basel, 1994.

[K-O] D. Kwon and Y.-G. Oh, Structure of the image of (pseudo)-holomorphic discs with totally real boundary condition. With an Appendix by Jean-Pierre Rosay, Communications in Analysis and Geometry 8 (2000), 31-82.

[La] L. Lazzarini, Existence of a somewhere injective pseudo-holomorphic disc, Geometric and Functional Analysis 10 (2000), 829-862.

[M] K. Mohnke, Lagrangian embeddings in the complement of symplectic hypersurfaces, Israel Journal of Mathematics 122 (2001), 177-123.

[M-S] D. McDuff and D. Salamon, Introduction to Symplectic Topology, Oxford University Press, 1995. 
[Oh-1] Y.-G. Oh, Removal of boundary singularities of pseudo-holomorphic curves with Lagrangian boundary conditions, Communications on Pure and Applied Mathematics 45 (1992), 121-139.

[Oh-2] Y.-G. Oh, Floer cohomology of Lagrangian intersections and pseudoholomorphic disks. I, Communications on Pure and Applied Mathematics 46 (1993), 949-993.

[Oh-3] Y.G. Oh, Floer cohomology, spectral sequences, and the Maslov class of Lagrangian embeddings, International Mathematics Research Notices (1996), No. 7, 305-346.

[Oh-4] Y.-G. Oh, Gromov-Floer theory and disjunction energy of compact Lagrangian embeddings, Mathematical Research Letters 4 (1997), 895-905.

[Oh-5] Y.-G. Oh, Relative Floer and quantum cohomology and the symplectic topology of Lagrangian submanifolds, in Contact and Symplectic Geometry (Cambridge, 1994), Publications of the Newton Institute, 8, Cambridge University Press, Cambridge, 1996, pp. 201-267.

[P] L. Polterovich, Monotone Lagrange submanifolds of linear spaces and the Maslov class in cotangent bundles, Mathematische Zeitschrift 207 (1991), 217-222.

[Sc] M. Schwarz, Morse Homology, Progress in Mathematics, 111, Birkhäuser Verlag, Basel, 1993.

[V-1] C. Viterbo, A new obstruction to embedding Lagrangian tori, Inventiones Mathematicae 100 (1990), 301-320.

[V-2] C. Viterbo, Functors and computations in Floer homology with applications. I, Geometric and Functional Analysis 9 (1999), 985-1033. 